the DEX, Neuropsychiatric Inventory (NPI), SF-36 and Zarit Burden Scale. Patient insight was measured using the discrepancy between carer and patient total scores on the DEX questionnaire.

Results: The DEX discrepancy score correlated significantly with total burden score $(r=.52$, $P=0.009)$. Burden was not correlated with patient cognition, age, neuropsychiatric symptoms and patient or carer SF-36 scores. A stepwise multiple regression with total burden as the outcome variable was statistically significant $\left(R^{2}=.65, \mathrm{~F}=8.72, P<0.001\right)$, significant predictors of outcome were DEX discrepancy, patient GDS, CDR sum of boxes and NPI score. Carer relationship and living status did not affect perceived burden.

Conclusions: Reduced insight in patients with dementia may result in increased isolation and frustration for their carer (compared with carers of patients who have good insight). Therefore, measuring insight in patients with dementia may be useful in identifying carers at risk for high burden levels.

\section{e-couch: the mother of all emental health web sites}

\section{K Griffiths, A Bennett, K Brittliffe, H Christensen}

Centre for Mental Health Research, ANU, Canberra, Australia

Background: Evidence from randomized controlled trials shows that Internet-based emental health selfhelp programs can improve mental health outcomes. However, these interventions are typically not tailored to a user's particular risk factors or specific symptoms and are often associated with high attrition rates.

Methods: This poster describes e-couch, a secondgeneration tailored cognitive behaviour therapy and information programme. e-couch provides information and automated self-help tools for depression, generalized anxiety disorder, social anxiety disorder, self-help for mental health problems associated with bereavement and divorce and separation, and a stigma-busting section. Other modules, including panic disorder, postnatal depression and physical illness, are in preparation. Tools include interpersonal therapy, cognitive therapy, exposure, social skills training, problem solving, applied relaxation, online exercise management, sleep hygiene and others. The system has been engineered to run automated online randomized controlled trials and to facilitate the addition of new streams tailored to particular at-risk groups and conditions.

Results: e-couch will be available for use by the public from January 2007. Initial research studies will investigate the effect of tailoring and automated monitoring on efficacy and retention. Outcome measure will include symptom levels, stigma, program adherence, knowledge and help seeking.

Conclusions: Key members of the team who developed e-couch will be available to answer questions and demonstrate the system.

Acknowledgement: e-couch (v1.0) was funded by beyondblue: the national depression initiative and the Centre for Mental Health Research, the Australian National University.

\section{Predictors of suicide in major depressive disorder: a follow-up of patients seen at a specialist mood disorders unit}

\section{Hadzi-Pavlovic', S Quinn', G Parker 1,2, P Mitchell', K Wilhelm'1', H Brodaty', G Malhi'2,3,4}

${ }^{1}$ School of Psychiatry, University of New South Wales, Sydney, Australia; ${ }^{2}$ The Black Dog Institute, Sydney, Australia; ${ }^{3}$ Academic Discipline of Psychological Medicine, Northern Clinical School, The University of Sydney, Australia; and

4Prince of Wales Medical Research Institute, Sydney, Australia

Background: Suicide is a major morbidity associated with depressive disorders. The Mood Disorders Unit (MDU), a specialized tertiary referral depression clinic, is currently undertaking a long-term follow-up of patients seen between 1985 and 1995 and diagnosed with major depression.

Methods: Death certificates and cause of death have been obtained for those in the cohort who have died. Cases of suicide will be compared with other subsets of the cohort using data from comprehensive assessments at the time of their index episode.

Results: The number who have died in the 10-20 years since their index episode from the initial cohort of $n=1008$ will be reported. Identifying cases of suicide is still being completed. Suicide rates and predictors of suicide in the cohort will be presented.

Conclusions: Variables predicting suicide 10-20 years later in a cohort of patients referred to a tertiary referral service will be discussed.

\section{Bipolar disorder and the $\mathrm{TCl}$}

\section{J Harley, P Joyce, E Wells, C Frampton \\ Christchurch School of Medicine and Health Sciences, New Zealand}

Background: Personality traits have been proposed as potential endophenotypes for genetic studies of psychiatric disorders. One personality theory that demonstrates strong heritability is Cloninger's seven-dimensional 